This item was submitted to Loughborough's Research Repository by the author.

Items in Figshare are protected by copyright, with all rights reserved, unless otherwise indicated.

\title{
Exploring equipment electrocardiogram mechanism for performance degradation monitoring in smart manufacturing
}

\author{
PLEASE CITE THE PUBLISHED VERSION
}

https://doi.org/10.1109/tmech.2020.2992328

\section{PUBLISHER}

Institute of Electrical and Electronics Engineers (IEEE)

\section{VERSION}

AM (Accepted Manuscript)

\section{PUBLISHER STATEMENT}

(c) 2020 IEEE. Personal use of this material is permitted. Permission from IEEE must be obtained for all other uses, in any current or future media, including reprinting/republishing this material for advertising or promotional purposes, creating new collective works, for resale or redistribution to servers or lists, or reuse of any copyrighted component of this work in other works.

\section{LICENCE}

\section{All Rights Reserved}

\section{REPOSITORY RECORD}

Chen, Baotong, Jiafu Wan, Min Xia, and Eve Zhang. 2020. "Exploring Equipment Electrocardiogram Mechanism for Performance Degradation Monitoring in Smart Manufacturing”. Loughborough University. https://hdl.handle.net/2134/14261138.v1. 


\title{
Exploring Equipment Electrocardiogram Mechanism for Performance Degradation Monitoring in Smart Manufacturing
}

\author{
Baotong Chen, Jiafu Wan, Member, IEEE, Min Xia, and Yu Zhang
}

\begin{abstract}
Similar to the use of ECG for monitoring heartbeat, this paper proposes an equipment electrocardiogram (EECG) mechanism based on fine-grained collection of data during the complete duration of operation of manufacturing equipment. The purpose of the EECG is to reveal the equipment performance degradation in smart manufacturing. First, this paper proposes system architecture of the EECG in smart manufacturing and explores the EECG mechanism including the granular division of the duration of the production process, matching strategy for process sequences, and several important working characteristics (e.g., baseline, tolerance and hotspot). Next, the automatic production line EECG (APL-EECG) is deployed to optimize the cycle time of the production process and to monitor the performance decay of the equipment online. Last, the performance of the APL-EECG was tested in the laboratory production line. the experimental results show that the APL-EECG can monitor the performance degradation of the equipment in real-time and improve the production efficiency of the production line. Compared with past FIS, APL-EECG had a more accurate and comprehensive understanding in terms of data of the production process. EECG mechanism contributes to both equipment fault tracking and optimization of production process. In the long run, APL-EECG can discover potential problems and provide guidance for the preventive maintenance of equipment.
\end{abstract}

Index Terms-EECG, performance degradation monitoring, production optimization, smart manufacturing

\section{INTRODUCTION}

A RITIFICIAL intelligence (AI) is the core driver behind highgrade transformation of manufacturing. With the rapid integration of manufacturing and information technologies, such as computing, communication and control, AI can facilitate the smart manufacturing system in obtaining the abilities of autonomous perception, abnormality detection, health management and active maintenance. The intelligent production line of industry 4.0 needs to meet the requirements

B. Chen and J. Wan are with the School of Mechanical and Automotive Engineering, South China University of Technology, Guangzhou 510641, China (e-mail: mecheney@mail.scut.edu.cn; mejwan@scut.edu.cn).

M. Xia is with the Department of Engineering, Lancaster University, United Kingdom (e-mail: m.xia3@lancaster.ac.uk).

Y. Zhang is with the Department of Aeronautical and Automotive Engineering, Loughborough University, United Kingdom (e-mail: Y.Zhang@lboro.ac.uk).

$\mathrm{J}$. Wan is the corresponding author. of multi-variety, small batch, and personalized customization production modes [1, 2]. Meanwhile, it puts forward stringent requirements for the safety, reliability and maintainability of the manufacturing equipment. However, the use of intelligent equipment will inevitably lead to performance degradation. Inspired by human electrocardiogram (ECG), we propose using equipment ECG called EECG to discover performance degradation rules for manufacturing equipment. As the equipment operates over fine-grained time slots, the goal of using the EECG is to monitor the performance degradation and optimize the cycle time of the production line. The EECG can discover potential problems and make the intelligent production line achieve preventive maintenance before an equipment fault actually occurs, and eventually form a closed-loop of "monitoring - diagnosis - maintenance".

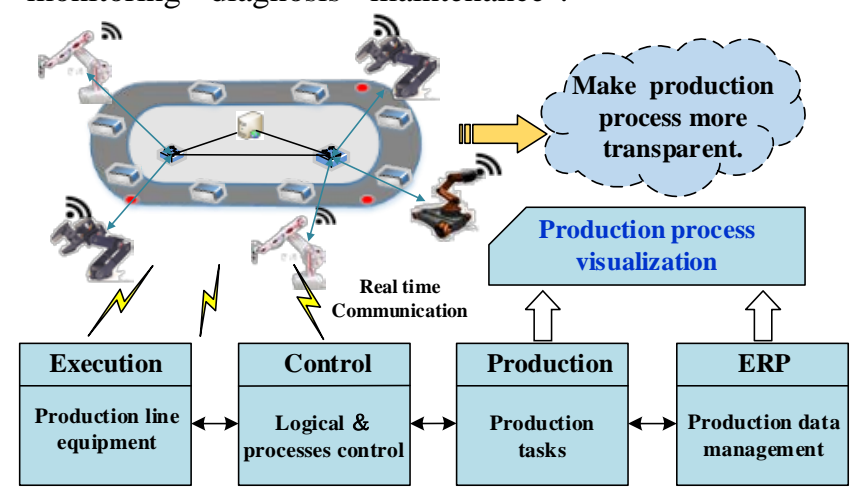

Fig. 1. Process visualization system model.

With the help of a mature data acquisition system, as shown in Fig. 1, the visualization of the production process contributes to making the production process more transparent. Archer et al. [3] proposed a task-oriented framework in data space for fine-grained data integration and data management, which is useful for storing fine-grained data and querying current states of the smart device. The ubiquitous industrial IoT makes it more convenient to collect, transfer and process the production data. As mathematical models and physical components of the intelligent equipment are highly complex, an early failure of complex equipment can still be predicted by data monitoring and rapid diagnosis $[4,5]$.

The development of the EECG can be divided into four stages: data collection, health index (HI) construction, health status monitoring and division, and prediction of remaining useful life [6]. Table I gives the definition for some related 
TABLE I. TERMINOLOGIES DEFINITION

\begin{tabular}{cl}
\hline Terminologies & \multicolumn{1}{c}{ Definition } \\
\hline Operation duration & $\begin{array}{l}\text { The length of time that manipulator or other } \\
\text { operation equipment lasts for completing } \\
\text { specific procedures. } \\
\text { The length of time that a production process } \\
\text { Process duration } \\
\text { lasts. The process duration can be division } \\
\text { several operation durations. } \\
\text { The length of time of a product repeatable } \\
\text { process lasts for a product or a product's part. } \\
\text { The noun can be applicated to production line } \\
\text { or workstation. } \\
\text { The time change that equipment is engaged in } \\
\text { or waiting for a task. It can be regarded as an } \\
\text { analogous to the heartbeat of EECG. } \\
\text { Production line beat is used to division the } \\
\text { spacing time and the working time of a } \\
\text { workstation for the whole production line. } \\
\text { Equipment (or workstation) beat can be } \\
\text { regarded as its elements. }\end{array}$ \\
\hline
\end{tabular}

terminologies. In [3], the framework guidance for automated data curation was given. Based on the continual basis, aggregated, and effectively mined, Abid et al. [7] proposed a model-based fault detection and isolation method, and the concept of fault severity index brings insight to identify the fault type and the severity level. Focusing on the dynamic slow decay process, Cao et al. [8] introduced the data-driven regression method to predict machining errors online. It should be noteworthy that the real-time performance of the prediction model restrains model complexity. Traore et al. [9] built an auto-adaptive dynamic classifier to track slow degradation for dynamical systems' components with inductive analysis methods. This work showed that failure prevention of a key component by processing of sensors data can avoid a long downtime of the system. Among them, sensors or other measuring devices are more accurate in certain aspects of health condition of the equipment. The emerging tendency raises the higher demands for the safety, reliability and maintainability of the manufacturing equipment. The performance degradation of the intelligent manufacturing equipment should be thoroughly analyzed. In this perspective, the operation duration may be a more comprehensive and more intuitive indicator to reflect the running state of the equipment.

Multi-component equipment monitoring using fine-grained data is an advanced method for predicting the remaining useful life of the equipment $[6,10]$. In [11], Rodrigues puts forward such a system to predict the remaining life based on performance indicators. This method relates the health factors of each component to the overall system performance. Prediction system of remaining useful life is performed by evaluating feature fitness by using deep neural network in [12]. The monotonicity and trend ability characteristics are closely related to $\mathrm{HI}$ and timeliness restrictions. The operation duration of the intelligent equipment is an important indicator of machine state. The fluctuation of the operation duration can effectively reflect the performance degradation process in real time. EECG system can provide a wide range of benefits to prediction of remaining useful life of equipment.
Computer software greatly promotes data-driven operation and maintenance mode in smart manufacturing [13]. Beet LLC built a process visibility system called enVision to provide realtime monitoring and process visualization for manufacturing equipment [14]. When the enVision system was applied to process visualization for a welding production line in a car plant, it proved that the system is effective in optimizing the cycle time by avoiding unreasonable programming, excessive welding time and some quality risks (e.g., explosive welding and gun sticking). Different the FIS (factory information system) that monitors the time from start to stop, enVision monitors the actual processes going on in between. However, the performance degradation monitoring of the manufacturing equipment is superficial by using the process visualization. Furthermore, unreasonable division of the production beat will also affect the efficiency of the production line. Romero-Silva et al. [15] pointed out that unbalanced average operation duration and buffer allocation would reduce the throughput of the production line. The extension work can be conducted that the duration of the processes and operations of the intelligent manipulator can be optimized by using mathematical planning methods, which is helpful to improve the cycle time of the intelligent production line.

Compared with the aforementioned existing works, the main contributions of this paper are as follows:

(1) Focusing on the performance degradation of the production line equipment, the EECG is built based on finegrained data collection during the whole operation duration, which is useful for monitoring the health status of the manufacturing equipment in real-time.

(2) The implementation mechanism of the EECG is explained in detail, including division of the complete process duration into fine-grained time slots, vector-improved dynamic time warping (DTW) algorithm for time-series process matching, and several important working characteristics such as baseline, tolerance, and hotspot.

(3) In terms of beat optimization of the intelligent production line and online monitoring of the equipment performance degradation, an optimization strategy of automatic production line EECG (APL-EECG) is proposed to improve the efficiency of the intelligent production line and help devise a preventive maintenance strategy for the intelligent equipment.

The paper is organized as follows. Section II proposes the system architecture of the EECG and its role in smart manufacturing. Section III presents the implementation mechanism of the EECG including granular division of the process duration, matching strategy for process sequences, and several important operating characteristics. Section IV introduces the EECG-based performance degradation monitoring and production optimization for intelligent production line. Section V implements the APL-EECG on a verification platform and analyses the experimental results. Section VI concludes this paper.

\section{SyStEM ARCHITECTURE}

The real-time acquisition of dynamic data information enables the EECG to monitor the operation status of an 


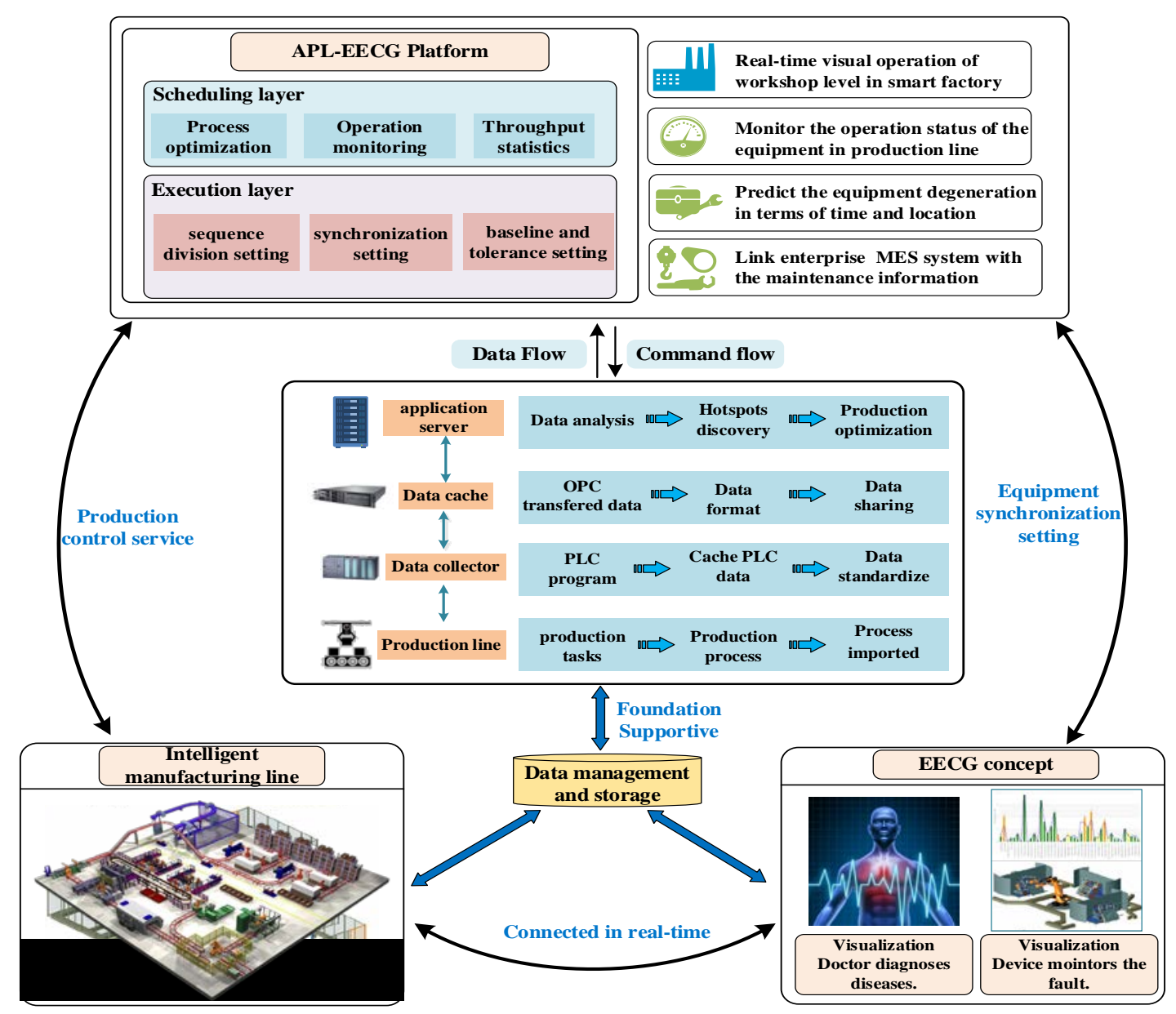

Fig.2. System architecture of EECG in smart factory

equipment and provide the time-series prediction of the equipment status. Fig. 2 shows the system architecture of the EECG for a mixed flow manufacturing production line in a discrete workshop. As shown in the figure, the architecture has three layers: First, the workshop automation network is utilized for data collection from the underlying equipment, including high-speed data collection by using PLC and OPC UA-based data unified transmission. Second, the data server is used for format management, data caching and data sharing. Last, the application server is deployed to provide the continuum analysis of the data, explore the hotspots and further improve the production cycle. The EECG represents an intuitive visual display of data, which contributes to providing an in-depth perception of the equipment status. To build the APL-EECG, it is crucial to develop novel techniques based on the following aspects: (1) scientifically divide the granularity of the manufacturing process; (2) choose the online matching algorithm for time-series process sequences; (3) set the baseline for a single equipment to complete a process or a task; and (4) determine the tolerance degree for the fluctuation of the operation time.

The platform of the APL-EECG includes scheduling and execution layers, which are used for setting the parameters and optimizing the production respectively. The EECG can clearly show the duration during which an equipment is in operation, monitor the operation status, reflect the degradation trend of the equipment performance, and provide strong support and a new mode for fault prediction for intelligent equipment. The APLEECG realizes real-time process visualization in the workshop and supports personalized customized production mode. With the operation status monitoring of the production line, the EECG can reveal the performance degradation and predict the evolution rule in terms of time, location and degree. In this way, when the EECG is linked to the PLM or MES, it can provide preventive maintenance guideline for the workshop equipment.

\section{EQUIPMENT ELECTROCARDIOGRAM MECHANISM}

The EECG monitors the operation status using the method of fine-grained processes visualization. Data acquisition from the underlying equipment forms the basis of the EECG. Fig. 3 shows the data acquisition processes in the APL-EECG. In discrete manufacturing, the production process of a workstation requires the equipment to complete the production task in a small amount of production time. The granularity division and fluctuation of the process duration are important EECG characteristics that can reflect a change in the equipment status. Considering these factors, this section focuses on the EECG mechanism including the method of multi-granularity division of the manufacturing process, matching strategy of the process sequence, and important characteristics of the APL-EECG. 


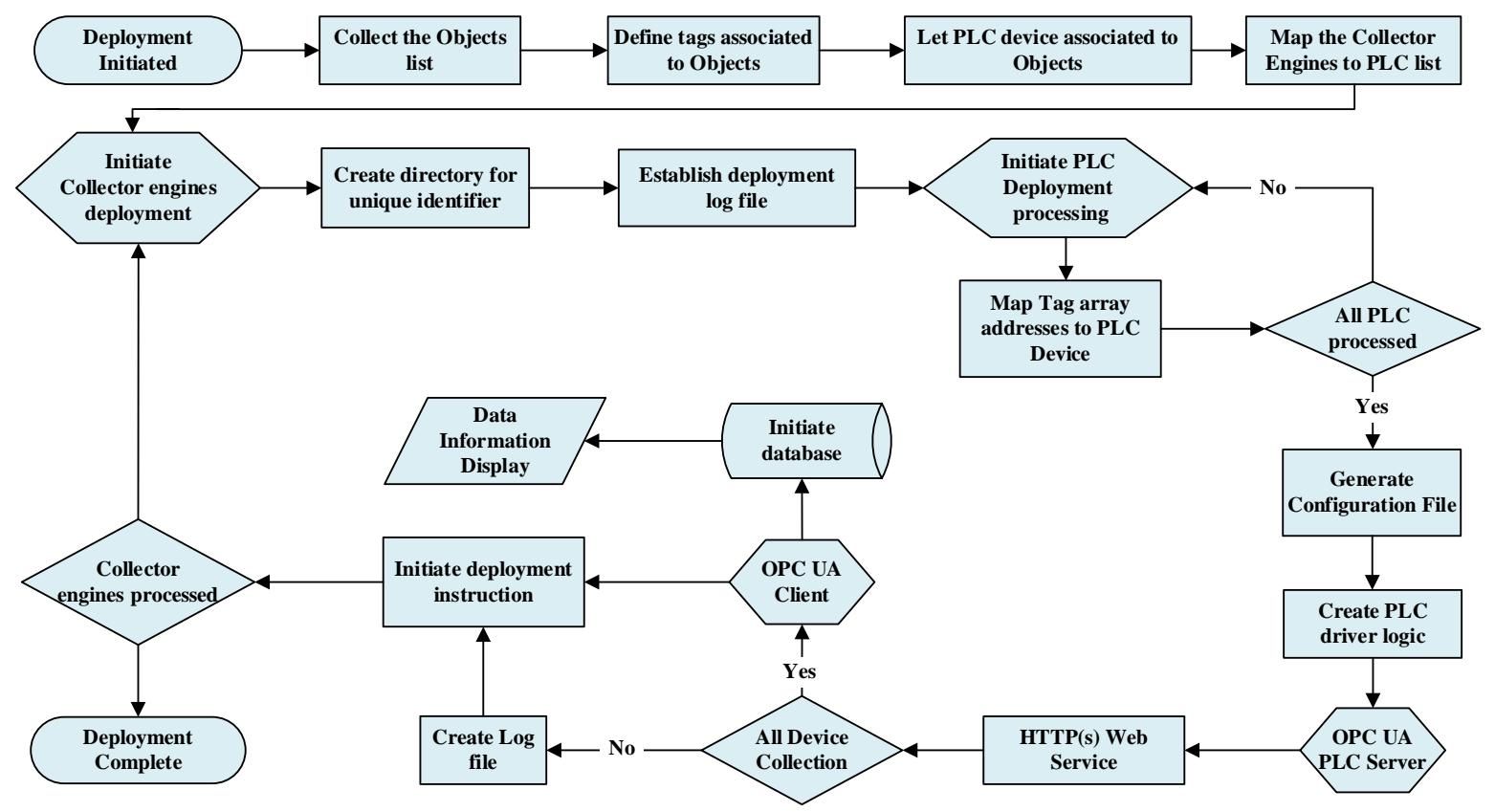

Fig. 3. Flow-chart of data acquisition in APL-EECG.

\section{A. Granular division of process duration}

The time length of the operation duration forms the basis for building the EECG. The product manufacturing process is often subdivided into multiple processing tasks. There may be many processing tasks between the start and stop of a station, and each processing task can be further subdivided into one or more sequences. The raw data collected by PLC or by the equipment itself is the operation duration with time series feature. Rough set theory has the formal definition for the information system (IS) [16]. When it comes to the data collection based on multigranularity, we were inspired by the rough set theory to define the EECG information system. In this sub-section, we introduce the multi granularity division method based on the rough set theory.

Definition 1: Representing the EECG information system for a single equipment by a four-tuple set $G R S=(U, P, V, f)$, the information system of the APL-EECG can be described as $M G R S=\left\{G R S_{i} \mid G R S_{i}=\left(U, P_{i},\left\{\left(V_{p}\right)_{p \in A T_{i}}, f_{i}\right\}\right)\right\}$.

In this system, $U=\left\{e_{1}, e_{2}, e_{3}, \ldots, e_{n}\right\}$ denotes the non-empty finite object set of the equipment function, also known as universe of the rough set. The process set denoted by $P=\left\{p_{1}, p_{2}, p_{3}, \ldots, p_{m}\right\}$ can be completed by the equipment function, while $V_{p}$ denotes the cycle length of the process with $V=\bigcup_{p \in P} V_{P}$. The function $f: U \times P \rightarrow V$ is a mapping information function, and $f(e, p) \in V_{p}$.

Definition 2: $\exists p_{i}(t) \in P \quad, \quad$ and $\forall e \in U$ constitute. $U=\left\{e_{i} \mid e_{i}(t)=\sum_{0 \leq i \leq m}\left(\sim \hat{p}_{i}(t)\right)\right\}$.

The cycle length of the operation duration for the ith process is denoted by $p_{i}(t)$. Based on the cycle length, the whole process is segmented, and the function of the equipment is divided into sequences of operations. The completion time of the equipment function depends on the total number of operation sequences.

Definition 3: Define that $f=\left\{f_{i} \mid f_{i}: U \times P_{i} \rightarrow V_{p_{i}}, \forall i \in \operatorname{div}(T)\right\}$ is a mapping function and the process set $P$ belongs to the function set $U$. The set $e=\left\langle\left(p_{1}, f\left(e, p_{1}\right)\right),\left(p_{2}, f\left(e, p_{2}\right)\right), \ldots,\left(p_{n}, f\left(e, p_{n}\right)\right)\right\rangle$ shows that the total time consumed by a function is composed of times consumed by different operations.

The operation and non-operation durations are divided according to the functional characteristics of the equipment. For non-continuous operation equipment, such as the empty stroke of the equipment, the change of time length can be effectively captured, and the signal will not be lost when the network transmission channel is highly utilized.

Definition 4: Assume that the total time consumed by an equipment during a manufacturing process is $X_{\text {total }}=\sum_{i=1}^{N} \sum_{j=1}^{M} f\left(x_{i j}\right)$, and the total operation duration is $E_{p}=\sum_{i=1}^{N} f\left(P_{i}\right)$, then the efficiency of the granularity division of the equipment process can be represented by $\mu_{p}=\ln \left(\frac{E_{p}}{X_{\text {total }}-E_{p}}\right)$, and the channel utilization of the transmission network can be represented by $\mu_{i}=\frac{1}{M N} \sum_{i=1}^{N} \sum_{j=1}^{M} x_{i j}$.

In the preceding expressions, $x_{i j}$ is the node of the operation division, and $\mu_{p}$ and $\mu_{i}$ are used to measure the granularity efficiency. The effective operation duration should be as long as possible according to the functional characteristics, so that $\mu_{p}>0$. To avoid increase in the network load, the action interval of the equipment completing the process is ignored after the process is divided, such as the reset action of the manipulator during assembly and the empty stroke of the welding manipulator during welding. 
The division of process duration is a complicated step. Definitions 1, 2 and 3 give a general method to represent the structure of the EECG information, and definition 4 gives a method to divide the process based on the functional characteristics of the equipment. The process division provides basic support for the determination of the subsequent cycle length.

\section{B. Matching strategy for process sequences}

The EECG system accurately reflects the process progress in real-time and meets the time critical requirements for anomaly detection. For an intelligent production line with variable mass customization, the product variety is changing in the running of production line. The process sequences for the different product will change in real-time. For the certain production categories, the processes sequences for the reference templates are collected in advance, and the reference templates are random selected from the collected process sequences. When the reference templates are known beforehand, and the desired process should be matched with the reference templates online.

To identify templates of different processes, dynamic matching of the process template and real-time sequence of the time-series signal needs to be carried out in the EECG system. Inspired by the distortion measure in audio matching, we know that DTW (Dynamic Time Warping) is applied to measure the similarity of two voice sequences, in which Euclidean Distance is used to calculate the distance between elements of sequences [17]. Process flow of intelligent production line is more complex. The elements of the matching sequence include station sequence and its process sequence. We use matrix to represent the two-dimensional matching sequence. It is difficult to realize two-dimensional sequence matching by using Euclidean Distance in the classical DTW method. Considering these factors, this sub-section introduces a vector improved DTW algorithm [18] for developing the matching strategy, which exploits the time-series characteristics of the manufacturing process.

The DTW is used to compare the similarity of two sequences. The main idea behind this algorithm is to provide the optimal path for nonlinear alignment by minimizing the accumulated distance between two similar sequences. Assume that $Q$ is the test template and $C$ is the reference template shown in (1).

$$
\left\{\begin{array}{l}
Q=q_{1}, q_{2}, \ldots, q_{i}, \ldots, q_{n} \\
C=c_{1}, c_{2}, \ldots, c_{j}, \ldots, c_{m}
\end{array} .\right.
$$

The DTW solves the time-series sequences correspondence problem between $Q$ and $C$ by using the time normalization function, as shown in (2) and (3).

$$
\begin{aligned}
& d_{i, j}=(x(i)-y(j))^{2} . \\
& D=\min _{C} \frac{\sum_{n=1}^{K}\left[d\left(x_{i(n)}, y_{j(n)}\right) \cdot w_{n}\right]}{\sum_{n=1}^{K} w_{n}} .
\end{aligned}
$$

In (3), $W=\left(w_{1}, w_{2}, \ldots, w_{k}, \ldots, w_{K}\right)$ denotes the warping path of the DTW. The minimum distance between the test and reference templates is given as $D$, which refers to the similarity of two sequences.

The time-series sequences of the EECG system meet the basic conditions of sequence matching by DTW, but these sequences have their particular features based on the production processes. Assume that a work piece in the whole production line is completed by $S$ workstations and each workstation is composed of one or at most $N$ processes. The mapping function $f: U \times P \rightarrow V$ denotes the operation duration, and when the number of the processes is less than $N$, the margin will be replaced by a zero value. The whole process is represented by a matrix of size $S \times N$, as shown in (4).

$$
F P=\left[\begin{array}{cccc}
f\left(e_{1}, p_{(1,1)}\right) & f\left(e_{1}, p_{(1,2)}\right) & \cdots & f\left(e_{1}, p_{(1, n)}\right) \\
f\left(e_{2}, p_{(2,1)}\right) & f\left(e_{2}, p_{(2,2)}\right) & \cdots & f\left(e_{2}, p_{(2, n)}\right) \\
\vdots & \vdots & \ddots & \vdots \\
f\left(e_{s}, p_{(s, 1)}\right) & f\left(e_{s}, p_{(s, 2)}\right) & \cdots & f\left(e_{s}, p_{(s, n)}\right)
\end{array}\right] .
$$

Let $\gamma_{i}=\left(f\left(e_{i}, p_{(i, 1)}\right), f\left(e_{i}, p_{(i, 2)}\right), \ldots, f\left(e_{i}, p_{(i, n)}\right)\right)$ as the $i$ th row of the above matrix, then (4) can be rewritten as $F P=\left[\gamma_{1}, \gamma_{2}, \ldots, \gamma_{i}, \ldots, \gamma_{s}\right]^{T}$. Subsequently, the production processes of the test template $F P_{\text {test }}$ and the reference template $F P_{\text {refer }}$ can be expressed as follows:

$$
\begin{aligned}
& F P_{\text {test }}=\left[\alpha_{1}, \alpha_{2}, \ldots, \alpha_{i}, \ldots, \alpha_{n}\right]^{T} \\
& F P_{\text {refer }}=\left[\beta_{1}, \beta_{2}, \ldots, \beta_{j}, \ldots, \beta_{m}\right]^{T} .
\end{aligned}
$$

From (5), the estimated distance between two components of the test and reference templates is $d_{i, j}=\left\|\alpha_{i}-\beta_{j}\right\|^{2}$, which is used to define a component in (6).

$$
D_{i, j}=d_{i, j}+\min \left\{\begin{array}{l}
D_{i, j-1} \\
D_{i-1, j} \quad(2 \leq i \leq M, 2 \leq j \leq N) \\
D_{i-1, j-1}
\end{array} .\right.
$$

The warping path $\phi_{(\alpha, \beta)}$ for the distance accumulation is determined by $\alpha_{i}$ and $\beta_{j}, \phi_{(\alpha, \beta)}(1)=(1,1)$ and $\phi_{(\alpha, \beta)}(K)=(M, N)$. The step-length $\psi(k)$ is subject to the constraint $0 \leq \psi(k)-\psi(k-1) \leq 1$.

The matching strategy for the process sequences can be summarized as follows. When the production sequences of the equipment are imported into the APL-EECG, the matching strategy is used for template identification. The distance between the test template and each template present in the template library is calculated. The shortest distance indicates the highest similarity of the production sequence. The most similar template is chosen for real-time alignment of the timeseries sequences. Algorithm 1 shows the vector improved DTW algorithm.

\section{Important operating characteristics}

The matching strategy for the process sequences ensures the alignment of sequence in the acquired time-series data. The length scales of the production sequences are needed to evaluate the acceptability of the operation duration. The baseline represents the expected or designed duration of each operation, 


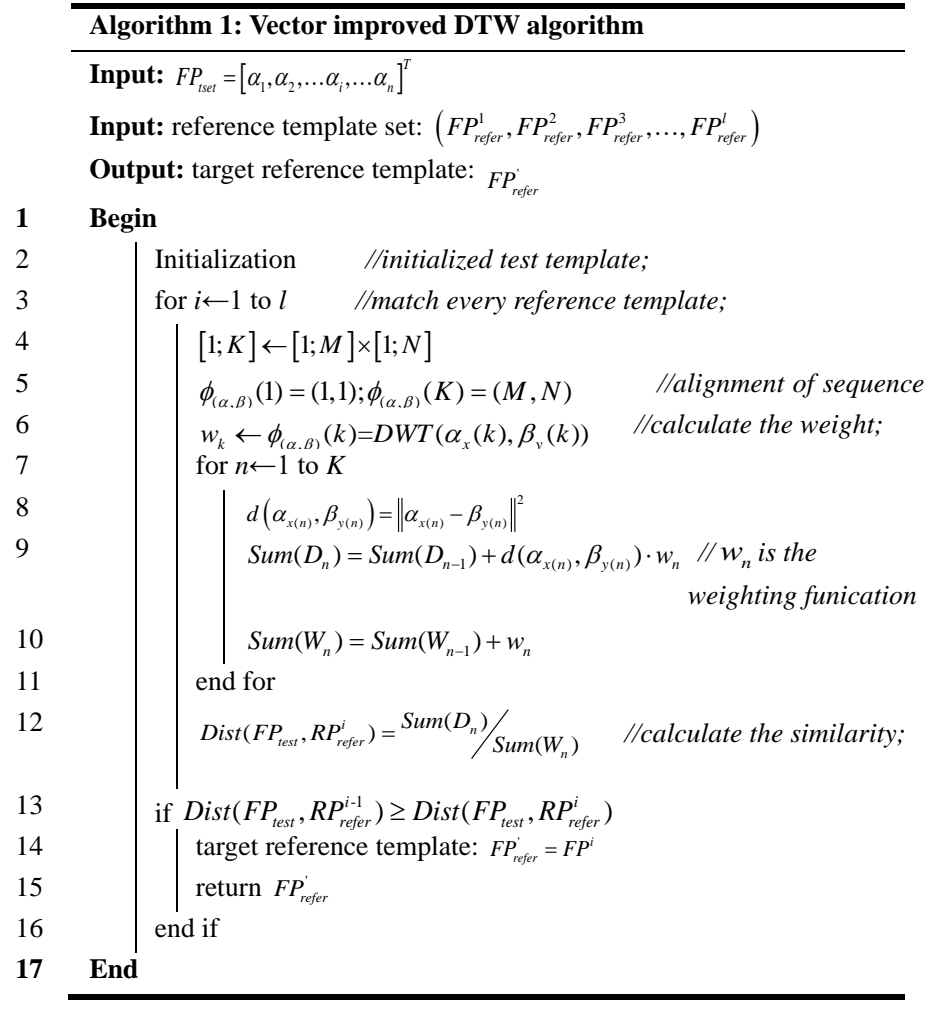

and the tolerance represents the floating value of the baseline after the baseline is determined. When the baseline and tolerance are set up, the operation duration is graded into either of the four levels: good, warning, warning, and fault. The determination of the baseline and tolerance is closely related to the time length of the operation duration. In this sub-section, the theoretical basis of these operating characteristics for the APLEECG is presented.

The baseline is used to demarcate the expected cycle length of the process, and it is also a base-value to measure the normality of the equipment beat. We use the sampling statistics to calculate the base-value of an operation duration. The root mean square (RMS) value of the sampling data is used as the base-value. Equation (7) reflects the cycle length of the sampling process of a random $N$ th times. The cycle length of the $i$ th sampling in the process $j$ is given by $p_{j}^{(i)}$, and $U_{B L}$ represents the base-value of a process in the EECG.

$$
U_{B L}=\sqrt{\frac{\sum_{i=1}^{N}\left[f\left(e, p_{j}^{(i)}\right)\right]^{2}}{N}} .
$$

Tolerance is closely related to the operation duration, which is also known as the beat. It is the benchmark for judging whether the equipment beat around the baseline is within a normal range. The modified sample variance is used to express the tolerance of the operation duration offset baseline as follows.

$$
S_{n}^{*}=\sqrt{\frac{1}{n-1} \sum_{i=1}^{n}\left(f\left(e, p_{j}^{(i)}\right)-U_{B L}\right)^{2}} .
$$

In (8), $S_{n}^{*}$ represents the tolerance value for fluctuation of the operation duration. The operation duration is graded according to the tolerance value. A serious deviation of a normally operating equipment from the baseline is an event with a small probability. According to the long-term sampling observations, the change of the operation duration follows a normal distribution. In this part, the $3 \sigma$ principle is used to evaluate the fluctuation of operation duration, and $\sigma \approx S_{n}^{*}$.

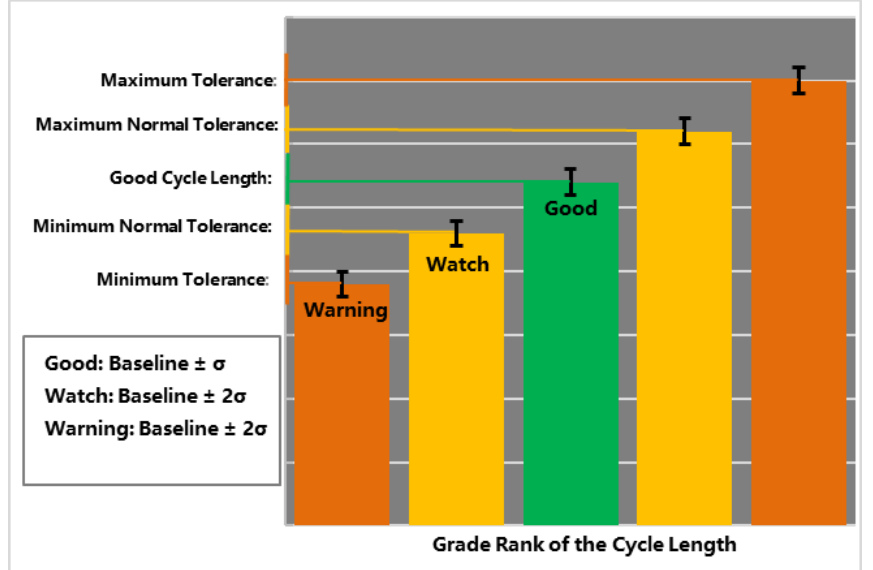

Fig. 4. Grading of the cycle length based on tolerance.

As shown in Fig. 4, the $\mathrm{X}$-axes reflect the graded for adjusting the values of cycle length. The Y-axis reflect the tolerance value based on the $3 \sigma$ principle. The grade division of $\mathrm{X}$-axis is followed the changing tolerance value of $\mathrm{Y}$-axis. When the operation duration is within the baseline tolerance, it will be marked as "good". The time length higher than the baseline tolerance will be marked as "watch", while that higher than the "nTol" value will be marked as "warning". When the operation duration is higher than the "Tol" value, it means that the equipment can malfunction.

In the APL-EECG system, the envelope is an important feature that reflects the floating range of the operation duration. It directly represents the extent to which the operation duration exceeds the baseline, and is calculated according to (9), where $U_{\text {Evp }}$ denotes the limiting value of the envelope. Under random sampling carried out $k$ times, $P_{J}=\left(p_{1}, p_{2}, p_{3}, \ldots, p_{J}\right)$, and $f\left(P_{J}\right)$ denotes the sampled value. In this paper, the higher limit of the envelope is adopted.

$$
\left\{\begin{array}{l}
U_{\text {Evp }}=U_{\text {BL }} \pm \min \sqrt{\sum_{m=1}^{k}\left(\frac{f\left(e, p_{j}^{(m)}\right)-f\left(P_{J}\right)}{f\left(P_{J}^{\text {(max) }}\right)-f\left(P_{J}^{(\text {min) }}\right)}\right)^{2}} \\
j \neq 1, j \in P_{J}
\end{array} .\right.
$$

\section{Performance Monitoring and Production OPTIMIZATION USING EECG}

\section{A. Automatic production line - EECG optimization of cycle} time

The cycle time mainly determines production rate of the intelligent production line. The operation performance of the equipment and logical settings of the operation instructions significantly impact the cycle time. In the welding production line of a car plant, the factors that can delay the equipment beat include inefficient waiting area, unreasonable interference area, loosening of clamping equipment, fluctuation of welding manipulator, and aging of equipment sensors. The APL-EECG can effectively reflect the change in operation duration and 
quickly determine the steps needed to improve the operation cycle time.

Using the APL-EECG, the method of mathematical programming is adapted to optimize the cycle time. When the equipment executes an external task, the collection of the equipment beat can be regarded as the sequence flow of the process, and the whole cycle time is composed of the work piece delivery time and the processing time. The operation duration will be reflected in the APL-EECG in a fine-grained fashion.

The mathematical optimization model of the cycle time in the APL-EECG is given in (10). The goal of this optimization is to minimize the operation duration and choose the appropriate conveying path of the work piece. It also makes the task execution sequence more compact when building the APLEECG.

$$
\begin{aligned}
& \min \quad \mathrm{Z}=\sum_{i=1}^{m} \sum_{j=1}^{m} x_{i j}\left(c_{i j}+o_{i j}\right) \\
& \text { s.t. }\left\{\begin{array}{l}
\sum_{i=1}^{m} x_{i j}=1, i=1,2, \ldots, m, \\
\sum_{j=1}^{m} x_{i j}=1, j=1,2, \ldots, m, \\
\sum_{i=1}^{s} \sum_{j=1}^{s} x_{i j} \leq s-1,2 \leq s \leq m-1, s \subseteq\{1,2, \ldots, \mathrm{m}\} \\
x_{i j} \in\{0,1\}, i, j=1,2, \ldots, m, i \neq j
\end{array}\right.
\end{aligned}
$$

In (10), the production task set is $T=\left\{t_{1}, t_{2}, \ldots, t_{m}\right\}$, and the operation instructions depends on the type of this task set. The transmission path of the work piece between stations $i$ and $j$ is denoted by $c_{i j}$, which is limited by the conveying path of the actual production line. The time consumed on the station is given by $o_{i j}$, and $x_{i j}$ denotes that task $t_{i}$ will be executed after the execution of task $t_{j}$, and also represents the direction in which the tasks continue. A global optimization algorithm used for solving (10) is given in algorithm 2 .

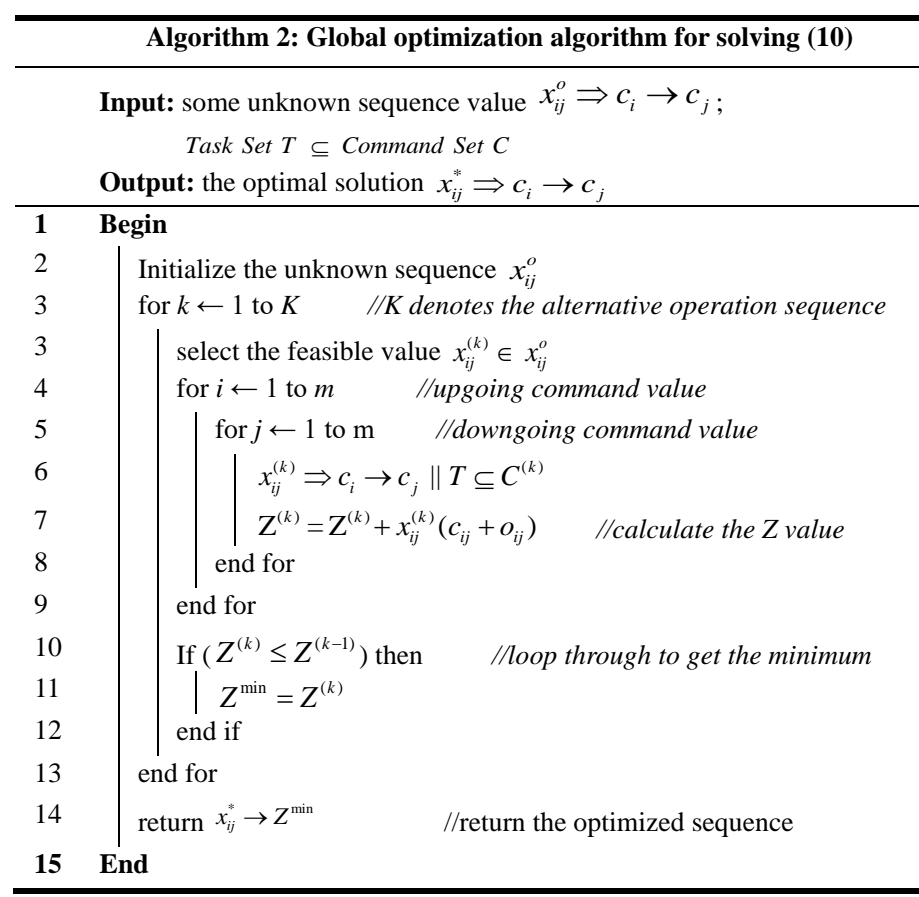

\section{B. Online monitoring of equipment performance decay}

Healthy operation of intelligent equipment is a guarantee for the highly-efficient production line.

traditional factory information system (FIS) has a single early-warning mode, which only records early warning events

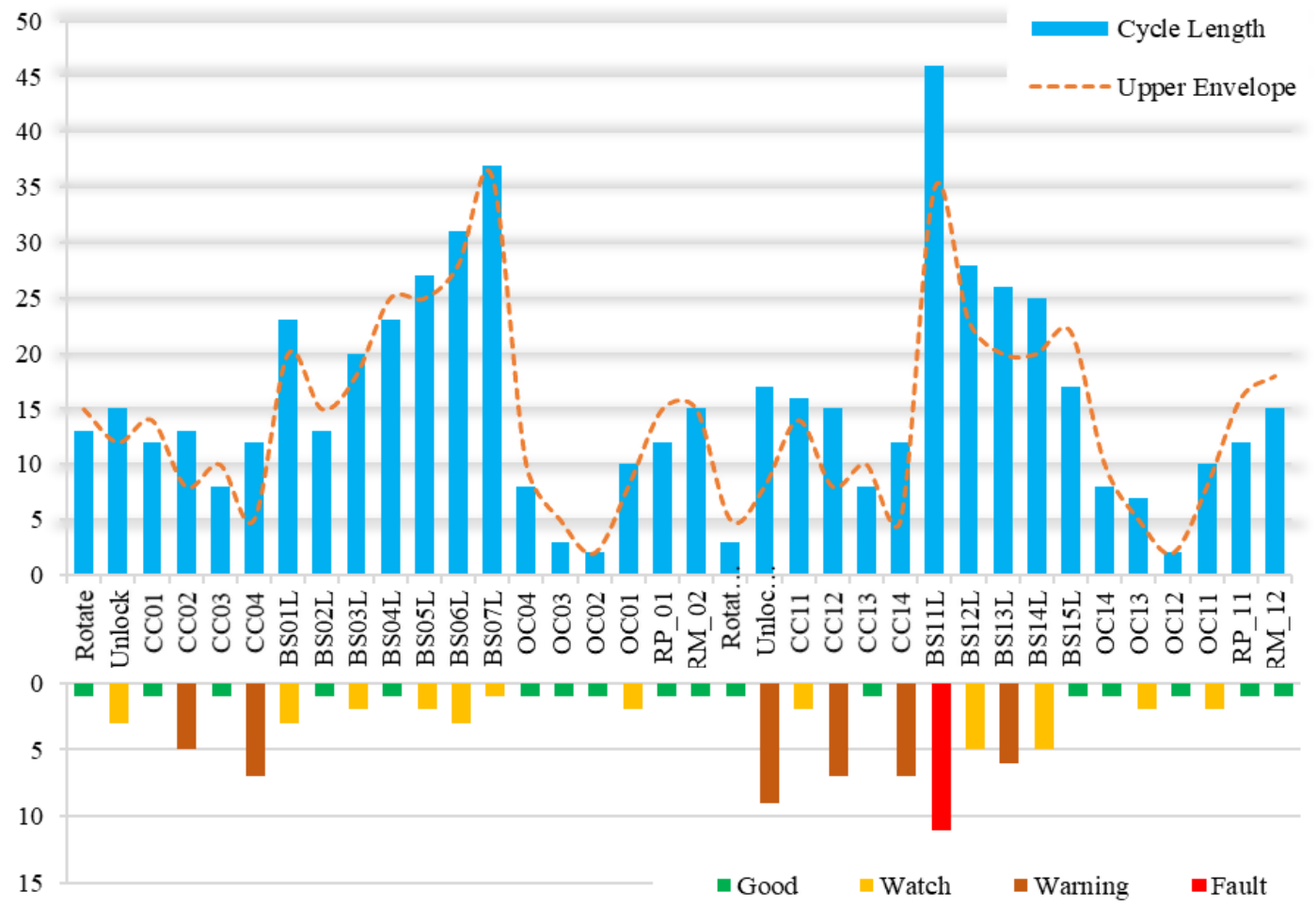

Fig. 5. Online monitoring of equipment performance decay. 
corresponding to a finite number of time nodes. Thus, losing a number of important event alarms is highly likely. The APLEECG system can continuously monitor the equipment performance decay and help predict the potential failure trend of the equipment visually. Changes in the continuous state are judged by the boundary condition. Deviation from the normal excessively or repeatedly will be marked as hotspots.

In terms of future performance degradation of the intelligent equipment, the APL-EECG adopts a grading scheme based on the emergency level. Fig. 5 reflect the online monitoring of equipment performance decay for several processes in the production line. The upper side of the horizontal axis shows the cycle length of a process, and the upper envelope gives the range of cycle length that can be floated upward. The lower side of the horizontal axis shows the grading level used for the cycle length evaluation. Those processes that are continuously graded as "warning" will be also labeled as hotspots. Hotspots are important references for developing effective preventive maintenance strategies for the equipment. During operation of production line, the performance of the equipment decays continuously. The online alarm mode of the APL-EECG can directly reflect the increase of the operation duration and accurately locate the source of an upcoming future performance degradation.

\section{IMPLEMENTATION OF EECG}

\section{A. Implementation scenarios}

An intelligent production line is built in our intelligent manufacturing laboratory, which involves workpiece carving and assembly, and patterns laser marking. The production line is a typical small batch discrete production line. The layout of the production line in the laboratory is shown in Fig. 6. The production stations are distributed discretely between the main line and the branch line, and RFID tags are used to store the operation information. The workpiece is transferred by the production line functions continuously.

In this sub-section, the implementation platform of the APLEECG is described, which was built based on our laboratory scenario. As shown in Fig. 6, every execution unit is deployed as the workstation of EECG, which can be divided into 13 sequences (SE) including nine manipulators, two CNCs, a marking machine, and an assembly machine. The inherent beat of each equipment is tested in advance because the operation times of different types of products vary greatly. Thereby, for testing purposes, the type of products processed by CNC or engraving machine is determined. The beat of the main working station is shown in Table II. The main validation schemes are listed as follows: (1) deployment of the APL-EECG; (2) improvement of the equipment beat; (3) performance degradation monitoring of the equipment; and (4) maintenance guidance for the production line.

TABLE II

BEAT OF THE MAIN WORKSTATIONS

\begin{tabular}{cccc}
\hline Workstation & Minimum beat & Average beat & Beat std \\
\hline Robot 1 & 2.3 & 3.5 & 1.7 \\
Robot 3 & 1.2 & 2.3 & 0.9 \\
Robot 5 & 2.4 & 3.7 & 1.3 \\
Robot 8 & 3.1 & 4.6 & 1.3 \\
Robot 9 & 3.2 & 5.1 & 1.6 \\
CNC-1 & 35.2 & 52.7 & 14.5 \\
CNC-2 & 32.7 & 50.6 & 15.3 \\
Assembly & 28.6 & 42.7 & 20.3 \\
Machine & & & \\
\hline
\end{tabular}

\section{B. Implementation results}

This sub-section shows the performance of the APL-EECG implementation based on the existing prototype platform. Tests were carried out over a duration lasting more than 90 days.

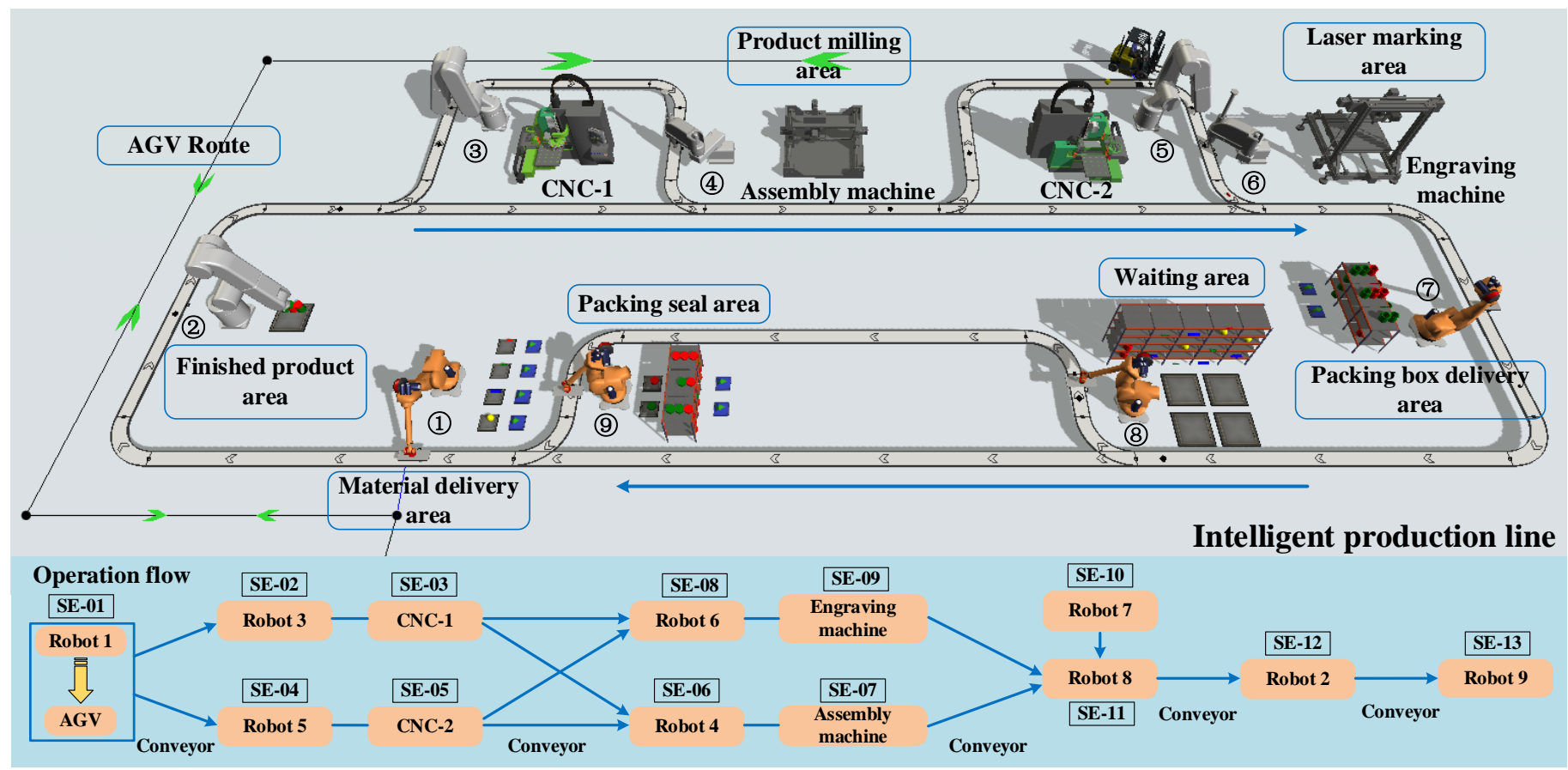

Fig. 6. Implementation platform of the APL-EECG in the manufacturing laboratory. 


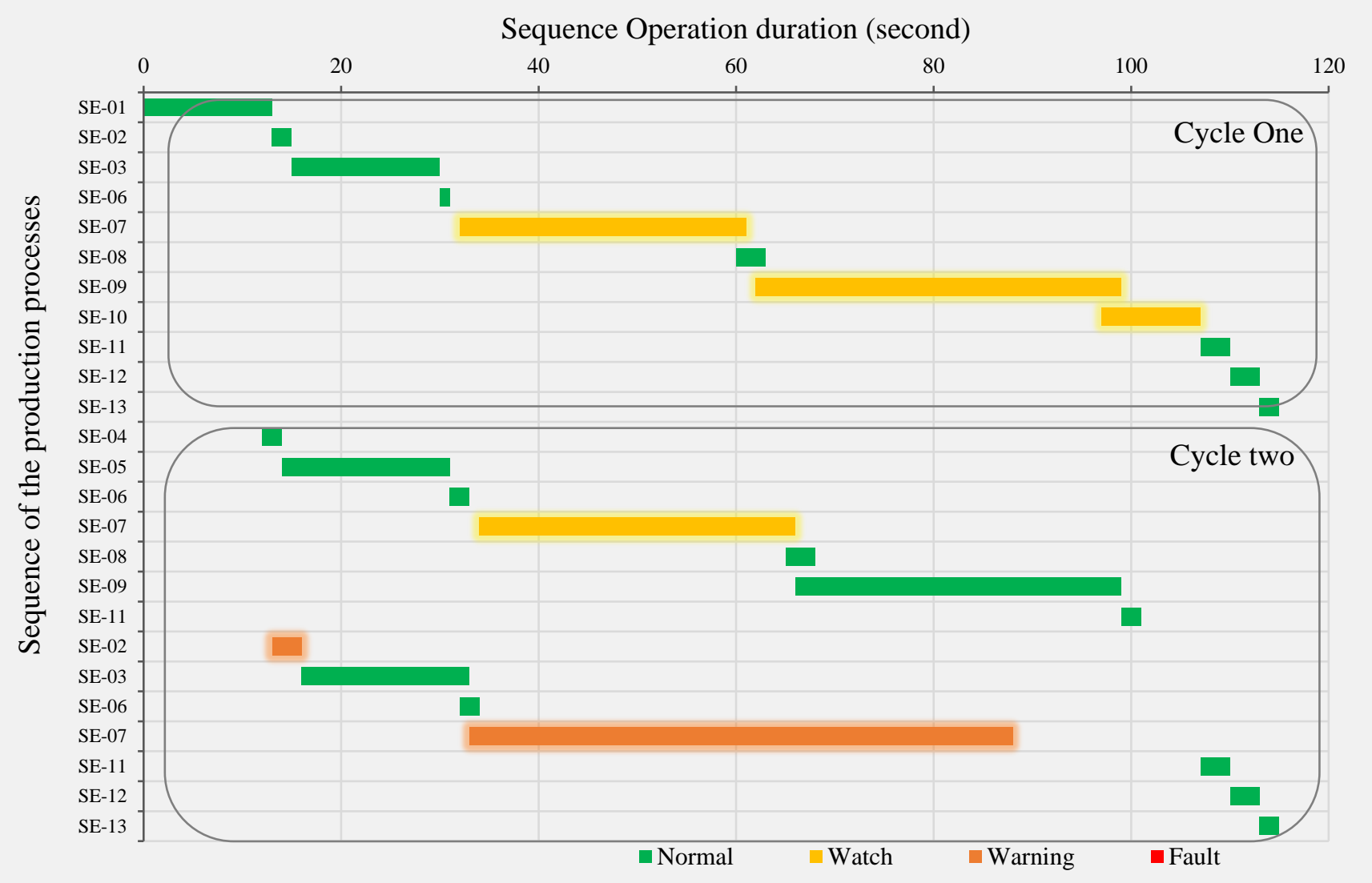

Fig. 7. Experimental results obtained from the APL-EECG platform.

Adjacent operation cycles showing large variation of cycle length are selected to reflect the production process of two different products, as shown in Fig. 7. The figure shows that the cycle length of the SE-07 increases continuously and exceeds the minimum tolerance range. Its grading information changes from "watch" to "warning", and consequently, its dynamic state will be displayed in the maintenance interface as a hotspot. The cycle length of SE-02 shows large fluctuations, which reflect the instability in its operation.
Table III lists parts of the alarm information of the APLEECG, which reflects the increase of the operation duration caused by aging equipment components. Based on the maintenance information of $S E-03$, its process changes from the "over cycle" and "blocked internally" to the final "blocked down" state. This demonstrates that the EECG platform can provide continuous monitoring information. Eventually it was discovered that the "blocked down" state was caused by the damage to manipulator caliper. As the traditional FIS is based

TABLE III

ALARM INFORMATION OF THE APL-EECG

\begin{tabular}{|c|c|c|c|c|c|c|}
\hline Time & Sequence & State & Duration & Status alert & Hotspot & $\begin{array}{l}\text { Maintenance } \\
\text { Assist }\end{array}$ \\
\hline Tues. 15:07:13 & SE-03 & Over Cycle & $46 \mathrm{~s}$ & Watch & No & No \\
\hline Tues. 16:08:43 & SE-02 & Over Cycle & $5 \mathrm{~s}$ & Watch & No & No \\
\hline Wed. 09:12:55 & SE-08 & Over Cycle & $7 \mathrm{~s}$ & Watch & No & No \\
\hline Wed. 11:23:15 & SE-03 & Internal Blocked & $52 \mathrm{~s}$ & Warning & Yes & No \\
\hline Wed. 15:09:20 & SE-04 & Over Cycle & $5 \mathrm{~s}$ & Watch & No & No \\
\hline Wed. 15:30:32 & SE-01 & Out of Auto & $53 \mathrm{~s}$ & Warning & Yes & Charging \\
\hline Wed. 17:27:08 & SE-09 & Over Cycle & $72 \mathrm{~s}$ & Warning & Yes & Tool Change \\
\hline Thur. 09:21:07 & SE-11 & Over Cycle & $18 \mathrm{~s}$ & Watch & No & No \\
\hline Thur. 10:14:32 & SE-02 & Over Cycle & $5 \mathrm{~s}$ & Watch & No & No \\
\hline $\begin{array}{r}\text { Thur. } \\
\text { 15:07:23 }\end{array}$ & SE-03 & Internal Blocked & $50 \mathrm{~s}$ & Warning & Yes & No \\
\hline Thur. 16:20:17 & SE-13 & Over Cycle & $8 \mathrm{~s}$ & Warning & Yes & No \\
\hline Fri. 10:23:31 & SE-01 & Over Cycle & 63 & Watch & No & No \\
\hline Fri. 13:35:12 & SE-04 & Internal Block & $12 \mathrm{~s}$ & Warning & Yes & Replace valve \\
\hline Fri. 15:42:55 & SE-03 & Blocked Down & $127 \mathrm{~s}$ & Fault & Yes & $\begin{array}{c}\text { Caliper } \\
\text { replacement }\end{array}$ \\
\hline Mon. 11:17:23 & SE-13 & Internal Blocked & $17 \mathrm{~s}$ & Warning & Yes & Fixture Change \\
\hline
\end{tabular}


on the timed feedback or manual operation, it is difficult to guarantee all-round relevance of the equipment status. The FIS does not reflect the decay process of the equipment but only its final status. On the contrary, the APL-EECG can provide continuous alarm information according to the fluctuation of the cycle length. Therefore, the APL-EECG can help in developing a reasonable maintenance strategy to avoid sudden "blocked down" state of the equipment.

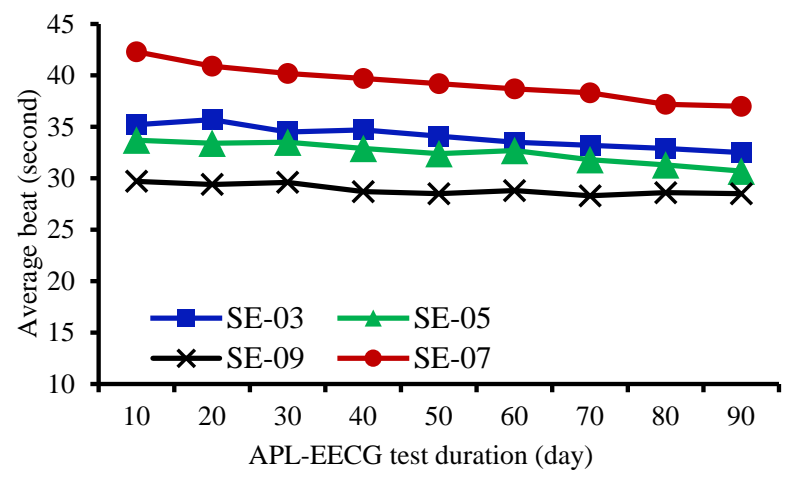

(a)

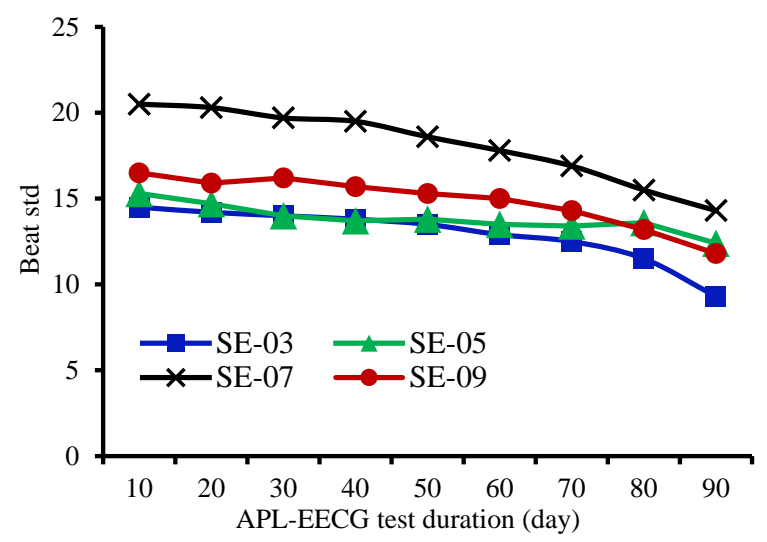

(b)

Fig. 8. Optimization effect of the APL-EECG on cycle time: (a) average beat and (b) beat std.

Based on Section IV.A, it can be seen that the deployment of the APL-EECG can provide the fine-grained operation status of the equipment and optimize the cycle time by using the mathematical programming methods. From the aspect of workstation, the delay can be reduced after the dwell time is reduced. In the early stage, the unreasonable program setting can be detected intuitively on the EECG panel. The aging components of equipment may increase dwell time during the operation. For example, if loosening and clamping of a part are checked simultaneously, tightening of the part can be accelerated, and the time between tightening and loosening can be reduced. From the aspect of mathematical planning, the information of workstation or manipulator that the action synchronization, program action and interference area can be optimized will feedback to the operation and maintenance system. Fig. 8 shows the optimization effect of the APL-EECG on the cycle time in our prototype platform. Fig. 8(a) demonstrates that the average operation duration of the four processes is gradually decreasing. From Fig. 8(b), it is evident that the standard deviation of the four processes is gradually decreasing, which indicates increasing stability of the equipment.

During the 90 days test, the number of times "over cycle" and "blocked down" occur in the APL-EECG system are counted. Under a typical FIS, the monitoring of production line would be limited to see when a cycle has started and stopped through the cycle time. APL-EECG monitors the time from start to stop, and monitors the actual processes going on in between. Compared with the FIS, Fig. 9 shows that the APL-EECG can effectively reduce both "over cycle" and "blocked down" counts, and the results show that the APL-EECG can improve the continuity of the intelligent production line.

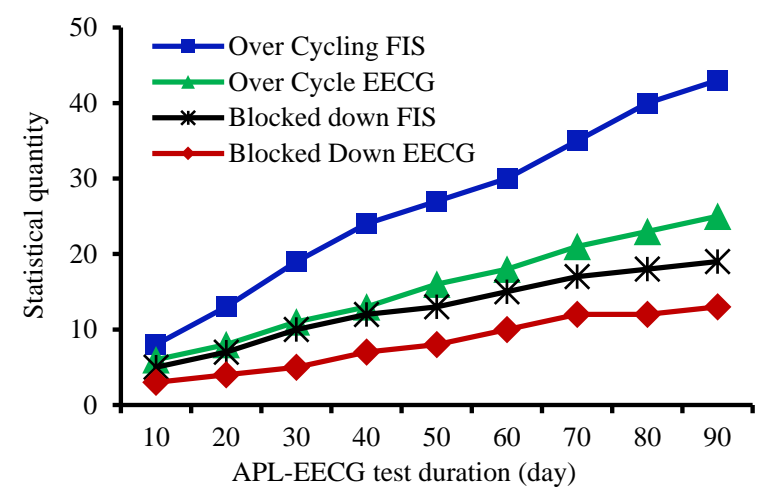

Fig. 9. Comparison of the performance of the FIS and APL-EECG over the complete test duration.

In the experimental results, we selected a wide range change of the cycle time to demonstrate the effect of implementing the APL-EECG. Practically, the cycle time of the production line is usually measured in seconds for automobile or packaging. Even a slight improvement of the equipment beat has the potential to increase the production line efficiency and bring huge economic benefits for enterprises. The equipment beat can be optimized by reducing the dwell time or modifying its unreasonable procedure settings. Considering the long life cycle of the equipment, the test on the equipment itself is not significant when most of the test data are normal. Because most intelligent equipment age gradually, the APL-EECG can effectively monitor the operation status of the equipment in the long run, and discover potential faults in the equipment.

\section{CONCLUSION}

The EECG presents the real-time operation status of the intelligent equipment visually. In terms of the performance degradation of the equipment, this paper explored the EECG mechanism for operational maintenance in an intelligent production line. The proposed APL-EECG can show the current equipment state, the state in the near-future, and ways of improving the state. A long-term test in laboratory settings showed that the APL-EECG system could monitor the operation status of the equipment in real-time and effectively improve the cycle time of the intelligent production line. The APL-EECG can help discover the performance imbalance of the manufacturing equipment in advance. Under laboratory conditions, the possibility of short-term equipment failure may 
be very small. In future, we will extend the fault prediction of the equipment and predict the remaining useful life using the EECG.

\section{REFERENCES}

[1] S. Wang, J. Wan, D. Li, and C. Zhang, "Implementing smart factory of industrie 4.0: an outlook," International Journal of Distributed Sensor Networks, vol. 12, no. 1, p. 3159805, 2016.

[2] J. Wan, S. Tang, D. Li, M. Imran, C. Zhang, C. Liu, et al., "Reconfigurable smart factory for drug packing in healthcare industry 4.0," IEEE transactions on industrial informatics, vol. 15, no. 1, pp. 507-516, 2018.

[3] D. W. Archer, L. M. Delcambre, and D. Maier, "A Framework for Finegrained Data Integration and Curation, with Provenance, in a Dataspace," in Workshop on the Theory and Practice of Provenance, 2009.

[4] Y. Merizalde, L. Hernández-Callejo, and O. Duque-Perez, "State of the art and trends in the monitoring, detection and diagnosis of failures in electric induction motors," Energies, vol. 10, no. 7, p. 1056, 2017.

[5] J. Wan, S. Tang, D. Li, S. Wang, C. Liu, H. Abbas, et al., "A manufacturing big data solution for active preventive maintenance," IEEE Transactions on Industrial Informatics, vol. 13, no. 4, pp. 2039-2047, 2017.

[6] Y. Lei, N. Li, L. Guo, N. Li, T. Yan, and J. Lin, "Machinery health prognostics: A systematic review from data acquisition to RUL prediction," Mechanical Systems and Signal Processing, vol. 104, pp. 799-834, 2018.

[7] A. Abid, M. T. Khan, H. Lang, and C. de Silva, "Adaptive System Identification and Severity Index-based Fault Diagnosis in Motors," IEEE/ASME Transactions on Mechatronics, 2019.

[8] L. Cao, X.-M. Zhang, T. Huang, and H. Ding, "Online monitoring machining errors of thin wall workpiece: a knowledge embedded sparse Bayesian regression approach," IEEE/ASME Transactions on Mechatronics, 2019.

[9] M. Traore, A. Chammas, and E. Duviella, "Supervision and prognosis architecture based on dynamical classification method for the predictive maintenance of dynamical evolving systems," Reliability Engineering \& System Safety, vol. 136, pp. 120-131, 2015.

[10] H. Yan, J. Wan, C. Zhang, S. Tang, Q. Hua, and Z. Wang, "Industrial big data analytics for prediction of remaining useful life based on deep learning," IEEE Access, vol. 6, pp. 17190-17197, 2018.

[11] L. R. Rodrigues, "Remaining useful life prediction for multiplecomponent systems based on a system-level performance indicator," IEEE/ASME Transactions on Mechatronics, vol. 23, no. 1, pp. 141-150, 2017.

[12] M. Xia, T. Li, T. Shu, J. Wan, C. W. De Silva, and Z. Wang, "A two-stage approach for the remaining useful life prediction of bearings using deep neural networks," IEEE Transactions on Industrial Informatics, vol. 15, no. 6, pp. 3703-3711, 2018.

[13] Y. Yuan, X. Tang, W. Zhou, W. Pan, X. Li, H.-T. Zhang, et al., "Data driven discovery of cyber physical systems," Nature communications, vol. 10, no. 1, pp. 1-9, 2019.

[14] https://resources.mongodb.com/migrating-to-mongodb/leaf-in-the-wildpowering-smart-factory-iot-with-mongodb. accesed

[15] R. Romero-Silva and S. Shaaban, "Influence of unbalanced operation time means and uneven buffer allocation on unreliable merging assembly line efficiency," International Journal of Production Research, vol. 57, no. 6, pp. 1645-1666, 2019.

[16] W. Wei and J. Liang, "Information fusion in rough set theory: An overview," Information Fusion, vol. 48, pp. 107-118, 2019.

[17] F. Petitjean, A. Ketterlin, and P. Gançarski, "A global averaging method for dynamic time warping, with applications to clustering," Pattern Recognition, vol. 44, no. 3, pp. 678-693, 2011.

[18] M. Morel, C. Achard, R. Kulpa, and S. Dubuisson, "Time-series averaging using constrained dynamic time warping with tolerance," Pattern Recognition, vol. 74, pp. 77-89, 2018.

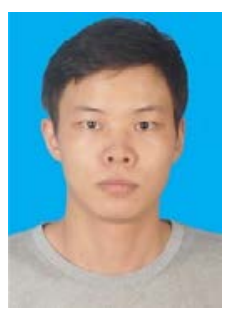

Baotong Chen is currently pursuing a Ph.D. degree in the School of Mechanical and Automotive Engineering at South China University of Technology (SCUT). His research interests include ML-enabled cognitive networks, edge computing in IIoT, data-driven active maintenance for smart equipment, and artificial intelligence applications in industry.

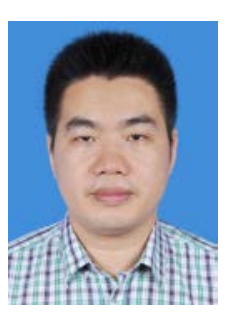

Jiafu Wan has been a Professor in School of Mechanical \& Automotive Engineering at South China University of Technology (SCUT) since Sep 2015. He joined in SCUT in May 2014. He received the Ph.D. degree in Mechatronic Engineering from SCUT in Jun 2008. His research interests include CyberPhysical Systems, Intelligent Manufacturing, Big Data Analytics, Industry 4.0, Smart Factory and Cloud Robotics.

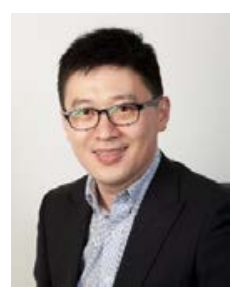

Min Xia is currently a lecturer in the Department of Engineering at the Lancaster University, UK. He received B.S. degree from Southeast University, China (2009); M.S. degree from the University of Science and Technology of China, China (2012); and Ph.D. degree from the University of British Columbia, Canada (2017). His research interests include smart manufacturing, machine diagnostics and prognostics, deep neural networks, wireless sensor network and sensor fusion.

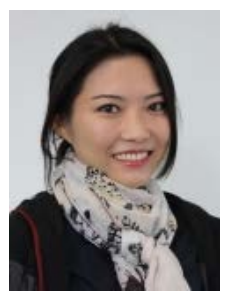

Yu (Eve) Zhang is a Senior Lecturer in Digital Engineering in the Department of Aeronautical and Automotive Engineering at Loughborough University since Sep. 2019. She obtained her BEng degree from the School of Aerospace Engineering and Applied Mechanics at Tongji University, China in 2004, and her $\mathrm{PhD}$ degree from the Department of Civil Engineering at the University of Nottingham in 2011. Her main research activities include condition monitoring, fault diagnosis and the development of data analysis and machine learning algorithms, especially for industrial applications. 